\title{
Reveal IP Spoofers Location from Route Backscatter and Passive IP Traceback
}

\author{
Akshay Dilip Homkar ${ }^{1}$, S. D. Satav ${ }^{2}$ \\ ${ }^{1}$ Department of Computer Engineering Jayawantrao Sawant College of Engineering, Hadapsar, Pune-28 \\ Savitribai Phule Pune University,Pune, India
}

${ }^{2}$ Professor, Department of Information Technology Jayawantrao Sawant College of Engineering, Hadapsar, Pune-28

Savitribai Phule Pune University,Pune, India

\begin{abstract}
Information science savoir-faire is used to hide the location s of the cyber-terrorist, spoofed. To identify the true spot of the spoofers Maturation of IP vestige back chemical mechanisms is used. Because of no common IP Traceback mechanism was adopted, Exact spoofers location was not identified till now. We implement Passive voice IP Traceback (Hell) mechanism to overcome the difficulties of the earlier technique itinerary backscatter messages (ICMP messages) generated by intermediate Synonyms/Hypernyms (Ordered by Estimated Frequency) of noun device in the network and traceback the spoofers using topology get detected by PIT. To identify the positioning of the spoofers, we apply Pit on path backscatter data set. the geographical location details of routing device near to IP spoofers are found, by employing the TTL field in IP packets.
\end{abstract}

Keywords: PIT (Passive IP Trackback), Computer Network Management, Computer Network Security, Denial of Service (DoS), IP traceback

\section{Introduction}

IP traceback is employed to construct the track traveled by info processing packets from provision to destination. A sensible and effective data processing traceback solution supported track disperse content, i.e., Infernal region, is program. PIT bypasses the readying difficulties of existing data processing traceback mechanism and real number ly is already effective. Though' given the limitation that route disperse substance don't seem to be geatomic number 10 rated with stable chance, PIT cannot ADHD all the attacks, however it will ADD variety of spoofing activities. a mini- mum of it should be the most helpful traceback mechanism before Associate in Nursing AS-level traceback organization has been deployed in real. Through applying PIT on the trail disperse dataset, variety of locations of spoofers lame measure captured and conferred. tho' this is often not a whole inclination, it's the 1st celebrated lean revealing the locations of spoofers. . PIT examines net direction Message Protocol blunder messages (named means backscatter) activated by mocking effort , and racecourse the spoofers in light-weight of open accessible information (e.g., regional anatomy ).Along these lines, PIT will notice the spoofers with no game arrange want. This paper represent to the explanations, accumulation, and therefore the authentic results on means disperse, exhibit the systems and adequacy of PIT, and shows the got regions of spoofers through applying PIT in transportation system disperse information set. These issue will assist additional with uncovering information processing spoofing, that has been examined for long but ne'er sure celebrated. In spite of the very fact that PIT cannot add all the spoofing attacks, it'd be the foremost valuable instrument to follow spoofers before Associate in Nursing Internet-level traceback framework has been sent in real.

\section{Review of Literature}

A. Castelucio, A. T. A. Gomes, A. Ziviani, and R. M. Salles 2012. In this paper, the crucial involvment of our proposal with respect to past work is its ability throughout a monitored network domain to provide partial and progressive deployment of the traceback system. The overlay network get built using the OSPF routing protocol through the creation of an IP Traceback Opaque LSA (Link State Advertisement) by us. Showing its suitability even for large network domains, We also investigate and evaluate the performance of partial and progressive deployment of the proposed system [1].

In the paper offered by M.-H. Yang and M.-C. Yang 2012 suggested a new hybrid IP traceback scheme with efficient packet logging. It is aiming to have a fixed storage requirement for each router in packet logging even without the need to refresh the logged tracking information and to achieve zero false positive and false negative rates in attack-path reconstruction. In addition, we utilize a packets marking field. We do so to censor attack traffic on its upstream routers. Finally,In evaluation with other related research, in the following aspects: computation, storage requirement, and accuracy, we simulate and analyze our scheme. [2].

M. Moreira, R. Laufer, N. Fernandes, and O. Duarte 2011. To allowing the victim to traceback the approximate origin of spoofed IP packets, we present two new schemes, the Advanced Marking Scheme and the Authenticated Marking Scheme. Our techniques support incremental deployment, feature low network and router overhead. Unlike previous work, our techniques have higher precision and lower computation overhead for the victim to reschedule the attack paths under large scale distributed denial of-service attacks. Furthermore even a compromised router cannot forge or tamper markings from other 


\section{International Journal of Science and Research (IJSR) \\ ISSN (Online): 2319-7064}

Index Copernicus Value (2015): 78.96 | Impact Factor (2015): 6.391

uncompromised routers, the Authenticated Marking Scheme provides efficient authentication of routers markings. [3].

C. Labovitz 2010. This paper proposes passive IP traceback (PIT). It totally sidesteps the sending challenges of IP traceback strategies. PIT examines Internet Control Message Protocol blunder messages (named way backscatter) activated by mocking movement. Also, tracks the spoofers in light of open accessible data (e.g., topology) too. On the same note, PIT can find the spoofers without any game plan. [4].

G. Yao, J. Bi, and Z. Zhou 2010. This article presents an Internet-scale Passive IP Trackback (PIT) mechanism. It does not require ISP deployment. as spoofed packets travel from attacker to victim, PIT analyzes the ICMP messages that may scattered to a network telescope. An Internet route model is then used to help re-construct the attack path. Cooperative Association for Internet Data Analysis (CAIDA) is applying this mechanism on the data collected by them, we found PIT can construct a trace tree from at least one intermediate router in 55.4Y. Xiang, W. Zhou, and M. Guo 2009. In this paper our main concentration on how packet marking is done as well as how we trace the source of attack. Now firstly the whole message is splits into multiple numbers of packets. According to marking Scheme algorithm, all Packets are marked on marker side. If intruder intrudes and gets access of the packets and modify them then with the help of reconstructor we reconstruct the same file at the receivers side. Finally receiver reconstructs the file and gets IP address of sender and hacker Using IP spoofing Technique, MAC address and Location of an intruder also. [6].

\section{System Architecture / System Overview}

This paper proposes Orchestra pit which is very different from any existing traceback mechanics. The briny difference of opinion is the propagation of track back scatter substance is not of a certain probability. Thus, we separate the evaluation into 3 piece : the first is the statistical resultant role $\mathrm{s}$ on route backscatter substance; the second is the evaluation on the traceback chemical mechanism offered in section IV-B without considering uncertainty of itinerary backscatter generation, since effectiveness of the mechanism is actually determined by the arrangement feature of the network ; the last is the result of performing the traceback apparatuses on the track backscatter message dataset. To avoid the challenge in deployment, We have proposed Passive Informatics Traceback (PIT). While sending an IP spoofing packet, there are multiple reason behind failing of router e.g., TTL exceeding. In such typeface, the router may garden truck an ICMP error message (named path backscatter). Meanwhile the source address get the distinction to the spoofed. Because the routers can be close to the spoofers. The path backscatter subject matter may get making water the positions of the spoofers. PIT exploits these path backscatter messages to find the position of the spoofers. With the positions of the spoofers known, the victim can seek help from the corresponding ISP to clean out the attacking mailboat, or take other counteroffensive. The victim in reflection based spoofing attacks, e.g., DNS amplification attacks get the benefit from PIT. The object from attacking traffic can find the area of the spoofers directly.

\section{System Analysis}

The architecture diagram of the system shown below helps us to understand the system. The mail boat s reach their

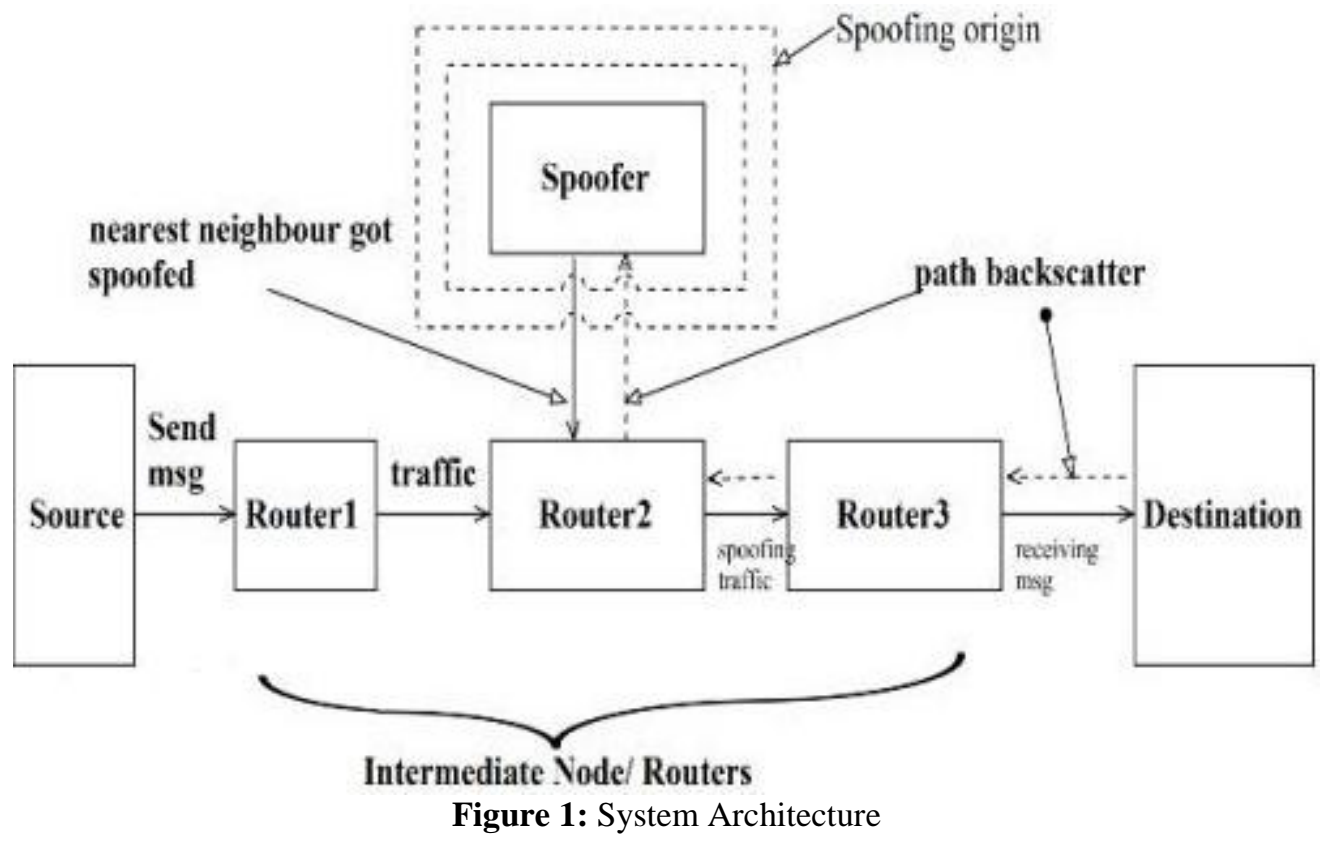

goals probably. While forward a packet network device may get failed due to some reason. It may produce an ICMP erroneous belief substance, i.e., track backscatter messages under certain shape. The route backscatter messages will be sent to the source Information science destination mentioned in the real packet. If the source name and savoirfaire gets forged, will be sent to the leaf node will accept the messages who is actually having the destination. It shows the victims of manifestation based attacks, as well as the hosts whose reference es are used by spoofers, are possibly 


\section{International Journal of Science and Research (IJSR) \\ ISSN (Online): 2319-7064 \\ Index Copernicus Value (2015): 78.96 | Impact Factor (2015): 6.391}

to collect such messages. This scenario is showed in Fig. I . Each and every message is having the source address of the reflecting device, as well as the Information science lintel of the master copy packet. Thus, from each pat backscatter, we can get 1) the Information processing address of the reflecting device which is on the path from the attacker to the destination of the spoofing packet; 2) the IP address of the original destination of the spoofing packet. The original IP header is having other valuable information, e.g., the remaining TTL of the spoofing packet. Distinction that due to some network devices may perform address rescript (e.g., NAT), the original source address as well as the destination address may be different in some cases.

\section{Mathematical Model}

\section{a) Set Theory}

Let $\mathrm{S}$ is the Whole System Consists: $\mathrm{S}=\mathrm{V}, \mathrm{E}, \mathrm{P}, \mathrm{G}$.

Where,

1. $\mathrm{V}$ is the set of all the network nodes.

2. $E$ is the set of all the links between the nodes in the network.

3. $\mathrm{P}$ is path function which defines the path between the two nodes.
4. Let $\mathrm{G}$ is a graph.

Suppose, G (V, E) from each path backscatter, the node $\mathrm{u}$, which generates the packet and the original destination $\mathrm{v}$, Where $\mathrm{u}$ and $\mathrm{v}$ are two nodes in the network.

We denote the location of the spoofer, i.e., the nearest router or the origin by s.

\section{b) Performance Analysis}

For performance measure we compare the actions perform of attackers IP of proposed system with the existing system actions. As in proposed system we are using IP blocking to prevent the future attacks. Hence we achieved the blocking action for our system. Because of this we can easily identify attackers and prevent future attacker by blocking the IP.

\section{c) Result Analysis}

Input:

Here, Existing System detect only IP with abnormal activity for the input purpose but here I mainly focuses on blocking IP of same system by which we are getting following result for our proposed system.

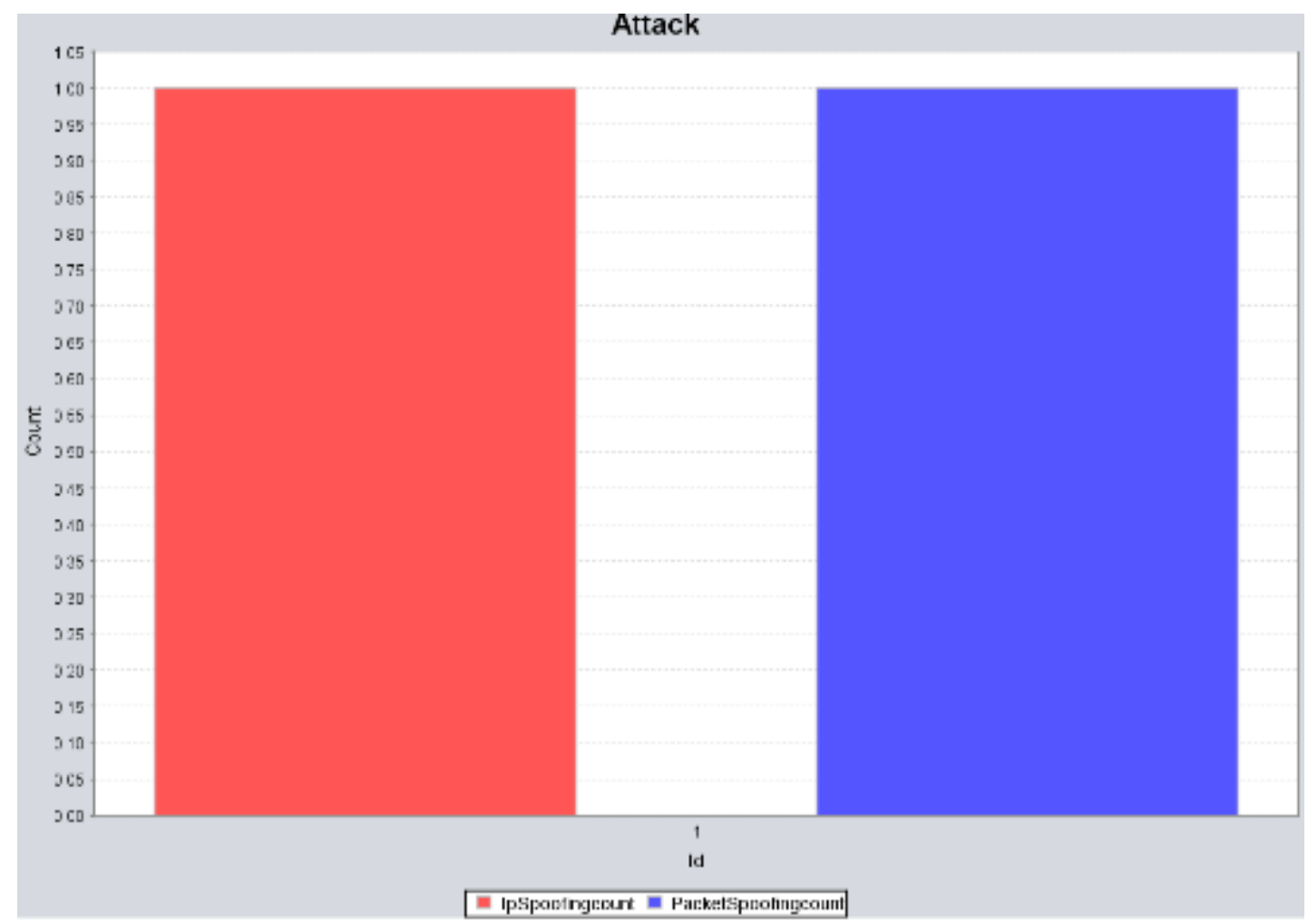

Figure 2: Expected Result Analysis

\section{Algorithm}

Particle Filtering based Algorithm

Particle filtering is a general Monte Carlo (sampling) method for performing inference in state-space models where the state of a system evolves in time and information about the state is obtained via noisy measurements made at each time step. In a general discrete-time state-space model, the state of a system evolves according to:

$$
\mathrm{xk}=\mathrm{fk}(\mathrm{xk} 1, \mathrm{vk} 1)
$$

where $\mathrm{xk}$ is a vector representing the state of the system at time $\mathrm{k}, \mathrm{vk} 1$ is the state noise vector, $\mathrm{fk}$ is a possibly non- linear and time-dependent function describing the evolution of the state vector. The state vector $\mathrm{xk}$ is assumed to be latent or unobservable. Information about $\mathrm{xk}$ is obtained only

through noisy measurements of it, zk, which are governed by the equation:

$$
\mathrm{zk}=\mathrm{hk}(\mathrm{xk}, \mathrm{nk})
$$




\section{International Journal of Science and Research (IJSR) \\ ISSN (Online): 2319-7064}

Index Copernicus Value (2015): 78.96 | Impact Factor (2015): 6.391

where $\mathrm{hk}$ is a possibly non-linear and time-dependent function describing the measurement process and $\mathrm{nk}$ is the measure- ment noise vector.

\section{Expected Result}

We proposing Passive IP Traceback (PIT) which tracks spoofers based on path backscatter messages and public available information. We specified how to apply PIT when the topology and routing are both known, or the routing is unknown, or neither of them are known. We presented two effective algorithms to apply PIT in large scale networks and going to proof their correctness. We are planning demonstrate the effectiveness of PIT based on deduction and simulation. We are going to show the captured locations of spoofers through applying PIT on the path backscatter dataset.

\section{Conclusion}

We proposed a method which is called as Passive voice Information science Traceback (Cavity). Perdition locate spoofers by taking help of path backscatter messages as well as public available information. We monitor causes, collecting, as well as statistical results on path backscatter. We clearly specified how to apply Nether region when the network topology as well as routing are both known in advance, or the routing is stranger, or neither of them are known. We introduced two effective algorithmic program to apply PIT in big network as well as proofed their accuracy. We demonstrated the effectiveness and perfection of PIT based on deductive reasoning as well as simulation. We also showed the tracked emplacement of spoofers by applying PIT on the path backscatter dataset. These results can and will help further reveal Information science spoofing, which has been subject for long but failed to get understand.

\section{Acknowledgment}

A successful work of this paper is the result of inspiration, support, guidance and cooperation of facilities provided during study. It gives me great pleasure to acknowledge my gratitude to present this paper titled: Reveal IP Spoofers Location from Route Backsatter and Passive IP Traceback. I place a deep sense of appreciation to my Project Guide Prof S D Satav and Prof. D R Patil giving me all possible help and suggestions to give this paper a perfect shape. I would like to thank all the authors of different research papers referred during writing this paper. It was very knowledge gaining and helpful for the further research to be done in future.

\section{References}

[1] Guang Yao, Jun Bi, Senior Member, IEEE, and Athanasios V. Vasilakos, Senior Member, IEEE Passive IP Traceback: Disclosing the Locations of IP Spoofers From Path Backscatter IEEE TRANSACTIONS ON INFORMATION FORENSICS AND SECURITY, VOL. 10, NO. 3, MARCH 2015
[2] S. M. Bellovin, Security problems in the tcp/ip protocol suite, SIGCOMM Comput. Commun. Rev., vol. 19, no. 2, pp. 3248, Apr. 1989.

[3] I. SSAC, Distributed denial of service (ddos) attacks, SSAC Adviso- rySAC008, Mar. 2006.

[4] A. Castelucio, A. T. A. Gomes, A. Ziviani, and R. M. Salles, IntradomainIP traceback using OSPF, Computer Communications, vol. 35, no. 5, pp. 554 564, 2012. [Online].

Available: http://www.sciencedirect.com/science/article/pii/S01403 66410003804

[5] M.-H. Yang and M.-C. Yang, Riht: A novel hybrid ip traceback scheme, Information Forensics and Security, IEEE Transactions on, vol. 7, no. 2, pp. 789797, April 2012.

[6] M. Moreira, R. Laufer, N. Fernandes, and O. Duarte, A stateless traceback technique for identifying the origin of attacks from a single packet, in Communications (ICC), 2011 IEEE International Conference on, June 2011, pp. 16.

[7] C. Labovitz, Bots, DDoS and Ground Truth, A presentation on NANOG 50th, Oct. 2010.

[8] G. Yao, J. Bi, and Z. Zhou, Passive ip traceback: Capturing the originof anonymous traffic through network telescopes, in Proceed- ings of the ACM SIGCOMM 2010 Conference, ser. SIGCOMM 10. New York, NY, USA: ACM, 2010, pp. 413414. [Online].

Available:http://doi.acm.org/10.1145/1851182.1851237

[9] Y. Xiang, W. Zhou, and M. Guo, Flexible deterministic packet marking: An ip traceback system to find the real source of attacks, Parallel and Distributed Systems, IEEE Transactions on, vol. 20, no. 4, pp. 567-580, 2009.

[10] D. Moore, C. Shannon, D. J. Brown, G. M. Voelker, and S. Sav- age,Inferring internet denial-of-service activity, ACM Trans. Comput. Syst., vol. 24, no. 2, pp. 115-139, May 2006. [Online]. Available http://doi.acm.org/10.1145/1132026.1132027.

[11]M. T. Goodrich, Efficient packet marking for largescale ip traceback, in Proceedings of the 9th ACM Conference on Computer and Commu- nications Security, ser. CCS 02. New York, NY, USA: ACM, 2002, pp. 117-126.

[12] J. Liu, Z.-J. Lee, and Y.-C. Chung, Dynamic probabilistic packet marking for efficient ip traceback, Computer Networks, vol. 51, no. 3, pp. 866 - 882, 2007.

[13]H. Wang, C. Jin, and K. G. Shin, Defense against spoofed ip traffic using hop-count filtering, IEEE/ACM Trans. Netw., vol. 15, no. 1, pp. 40-53, Feb. 2007.

[14] R. P. Laufer, P. B. Velloso, D. d. O. Cunha, I. M. Moraes, M. D. D. Bicudo, M. D. D. Moreira, and O. C. M. B. Duarte, Towards stateless single-packet ip traceback, in Proceedings of the 32Nd IEEE Conference on Local Computer Networks, ser. LCN 07. Washington, DC, USA: IEEE Computer Society, 2007, pp. 548-555. [Online]. Available: http://dx.doi.org/10.1109/LCN.2007.160

[15] The UCSD Network Telescope, http://www.caida.org/projects/ network telescope/ 


\section{International Journal of Science and Research (IJSR) \\ ISSN (Online): 2319-7064}

Index Copernicus Value (2015): 78.96 | Impact Factor (2015): 6.391

\section{Author Profile}

Mr. Akshay D. Homkar is currently pur suing M.E (Computer) from Department of Computer Engineering, Jayawantrao Sawant College of Engineering, Pune, India. Savitribai Phule Pune University, Pune, Maharashtra, India - 411007. He received his B.E (Information Technology) Degree from Padmabhooshan Vasantraodada Patil Institute of Technology, Budhgaon, India. Shivaji University, Kolhapur, Maharashtra, India - 416004. His area of interest is Network Security, IoT, Cloud Computing.

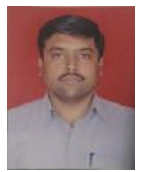

Assistant Professor Sandip Satav received the M.E (CSE/IT) degree from De partment of Computer Engineering, Vish wakarma Institute of Technology, Pune, MAH, India in 2004. He is currently work ing as Asst. Professor with Department of Information Technology, Jayawantrao Sawant. College of Engineering, Pune, $\mathrm{MAH}$, India. His research interests include Image Processing, Networking. 